13,05

\title{
Структурные и термоэлектрические свойства оптически прозрачных тонких пленок на основе одностенных углеродных нанотрубок
}

\author{
(C) И.А. Тамбасов ${ }^{1}$, А.С. Воронин ${ }^{2}$, Н.П. Евсевская ${ }^{3}$, М.Н. Волочаев ${ }^{1,4}$, Ю.В. Фадеев ${ }^{5}$, А.С. Крылов ${ }^{1}$, \\ А.С. Александровский ${ }^{1,5}$, А.В. Лукьяненко ${ }^{1,5}$, С.Р. Абелян ${ }^{1}$, Е.В. Тамбасова ${ }^{4}$ \\ ${ }^{1}$ Институт фризики им. Л.В. Киренского СО РАН, ФИЦ КНЦ СО РАН, \\ Красноярск, Россия \\ ${ }^{2}$ Красноярский научный центр, ФИЦ КНЦ СО РАН, \\ Красноярск, Россия \\ ${ }^{3}$ Институт химии и химической технологии СО РАН, ФИЦ КНЦ СО РАН, \\ Красноярск, Россия \\ ${ }^{4}$ Сибирский государственный университет науки и технологий им. М.Ф. Решетнева, \\ Красноярск, Россия \\ ${ }^{5}$ Сибирский фредеральный университет, \\ Красноярск, Россия \\ E-mail: tambasov_igor@mail.ru \\ (Поступила в Редакцию 2 апреля 2018 г. \\ В окончательной редакции 23 мая 2018 г.)
}

Тонкие пленки различной толщины из коммерчески доступных одностенных углеродных нанотрубок (ОУНТ) были изготовлены с помощью спрей-метода. Толщина ОУНТ-пленок была от $\sim 10$ до $\sim 80 \mathrm{~nm}$. Диаметр ОУНТ был в диапазоне значений $1.6-1.8 \mathrm{~nm}$. Было установлено, что существуют ОУНТ с длиной более $10 \mu \mathrm{m}$. Оптимальная толщина тонкой ОУНТ-пленки была $\sim 15 \mathrm{~nm}$ при которой пропускание было более $85 \%$. Удельное сопротивление тонких ОУНТ-пленок было от $\sim 1.5 \cdot 10^{-3}$ до $\sim 3 \cdot 10^{-3} \mathrm{Ohm} \cdot \mathrm{cm}$ при комнатной температуре. Впервые были исследованы температурные зависимости коэффициента Зеебека и поверхностного сопротивления для данного типа ОУНТ. Было обнаружено, что поверхностное сопротивление возрастает при увеличении температуры. Исследования показали, что тонкие ОУНТ-пленки имели слабую зависимость коэффициента Зеебека от температуры. Все образцы имели значение коэффициента Зеебека $\sim 40 \mu \mathrm{V} / \mathrm{K}$. Тонкие пленки показывали дырочный тип проводимости согласно знаку коэффициента Зеебека. Обнаружено, что фактор мощности $15 \mathrm{~nm}$ тонкой ОУНТ-пленки снижается при повышении температуры примерно до $140^{\circ} \mathrm{C} \mathrm{c} \sim 120$ до $\sim 60 \mu \mathrm{W} \cdot \mathrm{m}^{-1} \cdot \mathrm{K}^{-2}$. При дальнейшем увеличении температуры фактор мощности начинал увеличиваться.

Исследование выполнено за счет гранта Российского научного фонда (проект № 17-72-10079).

DOI: $10.21883 /$ FTT.2018.12.46739.086

\section{1. Введение}

Колоссальная часть неиспользованной тепловой энергии от промышленных производств и других областей жизнедеятельности людей рассеивается в окружающую среду. При этом часть этой тепловой энергии можно перевести в электрическую энергию с помощью термоэлектрических преобразователей. Стоит отметить, что самая значительная часть неиспользованной тепловой энергии находится в диапазоне температур до $200^{\circ} \mathrm{C}$ [1].

Для эффективного преобразования тепловой энергии в электрическую необходимы материалы с высокими термоэлектрическими свойствами. Ключевыми свойствами являются наличие высокой дырочной или электронной проводимости $\sigma\left(\mathrm{S} \cdot \mathrm{m}^{-1}\right)$, наличие высокого коэффициента Зеебека $S\left(\mathrm{~V} \cdot \mathrm{K}^{-1}\right)$, а также наименьшего коэффициента теплопроводности $\kappa\left(\mathrm{W} \cdot \mathrm{m}^{-1} \cdot \mathrm{K}^{-1}\right)$ в таких материалах [2]. Общей интегральной характеристикой термоэлектрических материалов является термоэлектрическая добротность $Z T=\left(S^{2} \sigma / \kappa\right) T$ где $T$ средняя температура между горячей и холодной сто- роной термоэлектрического преобразователя. Современные исследования, направленные на поиск и создания материалов с высокими термоэлектрическими характеристиками, сводятся к удовлетворению этих трех главных требований [2].

Безусловными лидерами по термоэлектрической добротности при температурах близких к комнатной являются соединения на основе висмута и теллура [3]. Для таких материалов термоэлектрическая добротность $Z T$ соответствует $\sim 1$ [3]. При этом, из таких соединений можно изготовить материалы с дырочной или электронной проводимостью. Однако главным недостатком таких материалов является использование токсичного теллура и постоянный рост цены на висмут и теллур.

В настоящее время сильное развитие получило направление гибкой электроники. В рамках этого направления стремительно развиваются работы по созданию и исследованию гибких термоэлектрических преобразователей [4,5]. Кроме этого, современная научная тенденция сейчас активно направлена на исследование и создание именно наноструктурированных термоэлектри- 
ческих материалов [6-9]. Это связано с тем, что за счет структурных особенностей можно изменять термоэлектрическую добротность $[7,9,10]$.

Ключевыми материалами для гибкой электроники являются различные аллотропные формы углерода. Касательно гибких термоэлектрических преобразователей, большую перспективу представляют полупроводниковые одностенные углеродные нанотрубки (ОУНТ) [11,12]. Это связано с тем, что в таких полупроводниковых одностенных углеродных нанотрубках был обнаружен высокий коэффициент Зеебека [12]. Кроме того, для достижения механической гибкости и эффективности термоэлектрического преобразования, создают и исследуют композитные термоэлектрические материалы на основе ОУНТ и традиционных термоэлектрических преобразователей.

В настоящее время прозрачные проводящие электроды на основе ОУНТ являются одним из основных кандидатов способных заменить прозрачные проводящие оксиды в оптоэлектронике [13]. Таким образом, существует возможность реализации нескольких функциональных свойств таких как электропроводность, высокая оптическая прозрачность и термоэлектрические свойства в рамках одного материала. Тонкие пленки ОУНТ, обладающие вышеперечисленными свойствами, могут быть использованы для прозрачной гибкой электроники, в качестве источников питания, позиционированных на прозрачном носителе [14].

Однако было найдено всего несколько публикаций посвященных исследованию термоэлектрических свойств тонких ОУНТ-пленок в температурном диапазоне выше комнатной. Стоит отметить, что в настоящее время налажено промышленное производство длинных ОУНТ с относительно низкой стоимостью. Таким образом, исследования термоэлектрических свойств тонких пленок из промышленно доступных ОУНТ является крайне актуальной задачей.

В данной работе впервые представлены исследования термоэлектрических свойств от комнатной температуры до $200^{\circ} \mathrm{C}$ оптически прозрачных тонких пленок на основе промышленно доступных ОУНТ.

\section{2. Методика получения и исследования пленок}

В работе использовался коммерчески доступный аэрогель ОУНТ (TUBALL, OCSiAl, Новосибирск), на основе которого приготавливались водные дисперсии. Полученные водные дисперсии с $0.1 \%$ массовой концентрацией ОУНТ использовались для изготовления тонких ОУНТ-пленок.

Тонкие пленки из ОУНТ осаждались с помощью спрей-метода на стеклянные подложки. Принцип формирования пленок ОУНТ заключался в следующем: сжатый воздух от компрессора подавался к аэрографу под давлением $0.6 \mathrm{MPa}$, распыляя дисперсию ОУНТ на нагретую подложку. Рабочая температура подложки составляла $130^{\circ} \mathrm{C}$. Нагрев подложки был необходим для того, чтобы исключить миграцию капель и предотвратить их коалесценцию. Средний размер капель аэрозоля составлял 30-50 $\mathrm{m}$. Расстояние от сопла аэрографа до подложки составляло $25 \mathrm{~cm}$. Толщина тонких пленок задавалась объемом распыленной дисперсии ОУНТ.

Контактные серебряные площадки были изготовлены с помощью вакуумной установки k575x Emitech. Толщина контактов была $\sim 500$ нм. Морфология поверхности тонких пленок определялась при помощи атомно-силового микроскопа DPN 5000 производства Nanoink. Для оценки толщины тонких ОУНТ-пленок использовался просвечивающий электронный микроскоп Hitachi TM7700 в режиме съемки поперечного сечения $[15,16]$.

Оптические свойства тонких ОУНТ-пленок исследовались при помощи спектрометра Shimadzu UV-3600 и спектрометра комбинационного рассеяния света Horiba Jobin Yvon T64000.

Измерения термоэлектрических свойств (коэффициента Зеебека и электрического сопротивления) тонких пленок от комнатной температуры до $200^{\circ} \mathrm{C}$ были проведены на установке собственной разработки. Разработанная установка состоит из термостата, прецизионного источника тока Keithley 2400, специально разработанного держателя для образца (задает градиентный нагрев образца), двух термопар $\mathrm{K}$ типа, а также 24-битного аналогово-цифрового преобразователя LTR114 на базе крейта LTR-EU-2 фирмы „Л Кард“ (Россия).

Изначально образцы тонких ОУНТ-пленок были прямоугольными с размерами $33 \times 25 \mathrm{~mm}$. Далее наносились две серебряные контактные площадки с шириной 4 и длиной $25 \mathrm{~mm}$ через маску с помощью магнетронного напыления. В результате получалась квадратная пленка ОУНТ с размерами $25 \times 25 \mathrm{~mm}$ для измерения термоэлектрических свойств. Для измерения электрического сопротивления использовалась двух зондовая схема. Два прижимных зонда с серебреными и сглаженными концами были расположены по одному на каждой серебряной контактной площадки образца. Измеряемый образец тонкой ОУНТ-пленки располагался на круглом сплошном медном столике с диаметром 42 и толщиной $2 \mathrm{~mm}$. Медный столик крепился в термостат Specac, через который происходил непрерывный нагрев. Скорость нагрева была $\sim 5 \mathrm{~K} / \mathrm{min}$. Непрерывный нагрев был близок к линейному от времени. Для контроля температуры использовались две рядом находящиеся термопары, расположенные на поверхности образца. Значение температуры принималось как среднее от температур, полученных от двух термопар. Для измерения сопротивления от температуры через контактные площадки образца пропускался прецизионный стабилизированный ток $I$ равный $50 \mu \mathrm{A}$ при помощи источника тока Keithley 2400. С этих же контактных площадок измерялось напряжение $V$ при помощи 24битного аналогово-цифрового преобразователя LTR114 на базе крейта LTR-EU-2. Сопротивление определялось как $V / I$. Кроме этого, данный аналогово-цифровой 


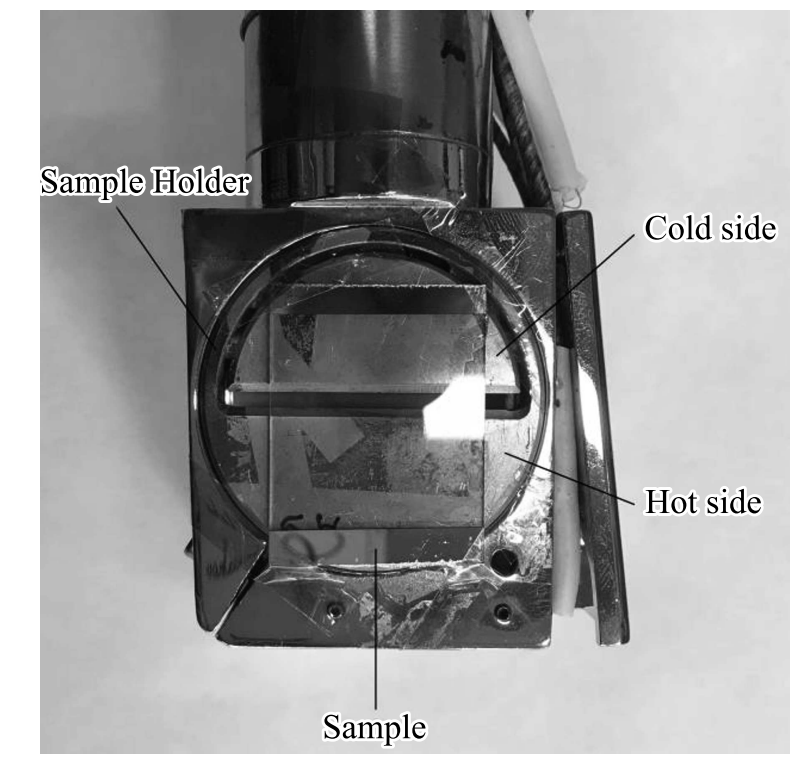

$a$

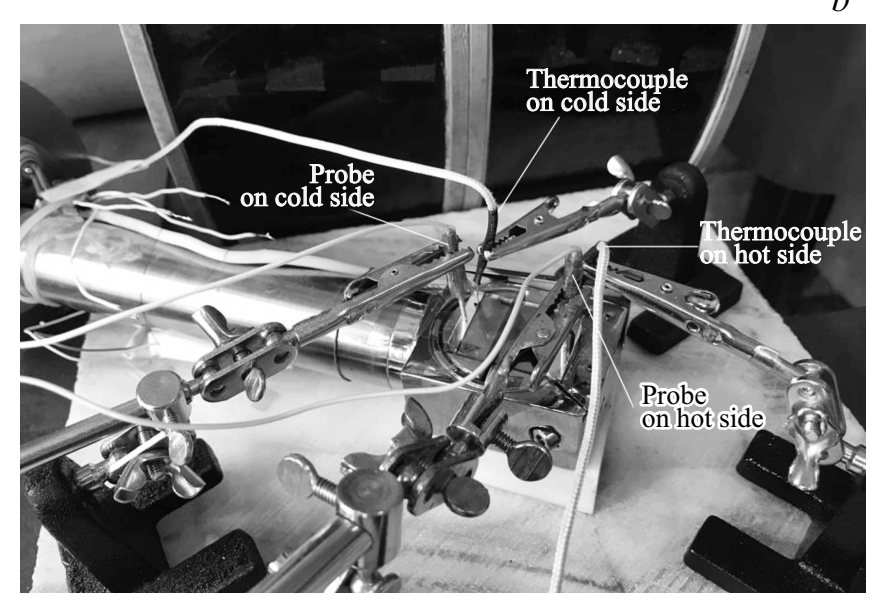

Рис. 1. Изображение держателя с образцов $(a)$ и общий вид подготовленного образца для измерения коэффициента Зеебека $(b)$.

преобразователь производил одновременно измерения с двух термопар. При измерении коэффициента Зеебека использовался специально разработанный держатель (рис. $1, a, b)$. При возникновении градиента температур при непрерывном нагреве держателя возникало электрическое напряжение $V_{3}$ на контактных площадках образца. Коэффициент Зебеека рассчитывался как $V_{3} / \Delta T$, где $\Delta T$ являлась разницей между температурами горячей и холодной стороны соответственно. Контроль и запись экспериментальных данных электрического сопротивления и коэффициента Зеебека проводились при помощи разработанного программного обеспечения.

\section{3. Экспериментальные результаты и обсуждение}

Для нахождения оптимального соотношения между оптическими и термоэлектрическими свойствами нами были изготовлены образцы тонких пленок из ОУНТ с различной толщиной. Толщина тонких пленок была $\sim 10, \sim 15, \sim 30, \sim 45$ и $\sim 80 \mathrm{~nm}$.

Известно, что комбинационное рассеяние света (КРС) является достаточно эффективным методом для исследования ОУНТ с точки зрения структурных и электронных свойств [17-19]. По этой причине мы использовали КРС для идентификации полученных тонких ОУНТ-пленок с наименьшей и наибольшей толщиной. На рис. 2 представлен спектр комбинационного рассеяния света для двух образцов тонких ОУНТ-пленок с толщиной 10 и $80 \mathrm{~nm}$.

Как видно из рис. 2 основные линии для 10 и $80 \mathrm{~nm}$ тонкой ОУНТ-пленки совпадают. Согласно линиям спектра КРС, представленным на рис. 1, тонкие пленки состоят именно из одностенных углеродных нанотрубок $[18,20]$.

Информативными для структурного анализа являются линии дыхательной моды RBM $\left(154 \mathrm{~cm}^{-1}\right)$ и $R 4 B M 2$ $\left(183 \mathrm{~cm}^{-1}\right)$. Здесь стоит отметить, что отдельные ОУНТ имеют одну дыхательную моду. Однако взаимодействие ОУНТ между собой в пучке приводит к появлению двух гибридных дыхательных мод вследствие понижения симметрии [18]. Такое взаимодействие существует в тонких ОУНТ-пленках. Согласно зависимости, представленной в работе [21], можно оценить диапазон диаметров ОУНТ в зависимости от значения гибридных дыхательных мод $R B M 1\left(154 \mathrm{~cm}^{-1}\right)$ и $R B M 2\left(183 \mathrm{~cm}^{-1}\right)$. Для наших тонких пленок диапазон диаметров ОУНТ был равен от $\sim 1.6$ до $\sim 1.8 \mathrm{~nm}$. С другой стороны диаметр ОУНТ можно оценить через следующее соотношение (1) [22]

$$
\omega_{R B M}=\frac{c_{1}}{d}+c_{2},
$$

где $\omega_{R B M}$ является дыхательной модой изолированной ОУНТ, $c_{1}$ и $c_{2}$ - поправочные коэффициенты, а $d-$ диаметр ОУНТ. Согласно работе [21], гибридная дыхательная мода RBM1 смещена относительно дыхатель-

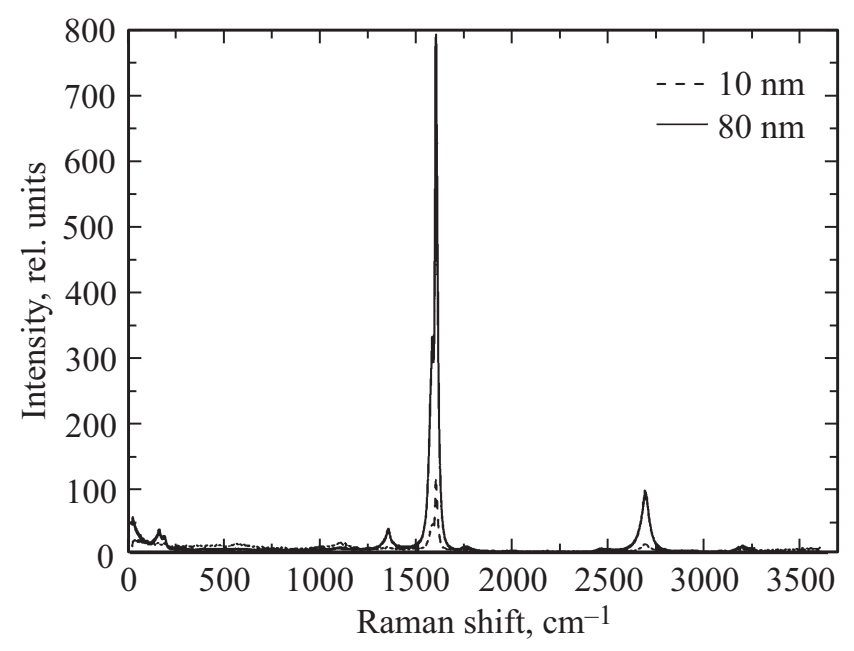

Рис. 2. Спектр комбинационного рассеяния света для 10 и $80 \mathrm{~nm}$ тонкой ОУНТ-пленки. 

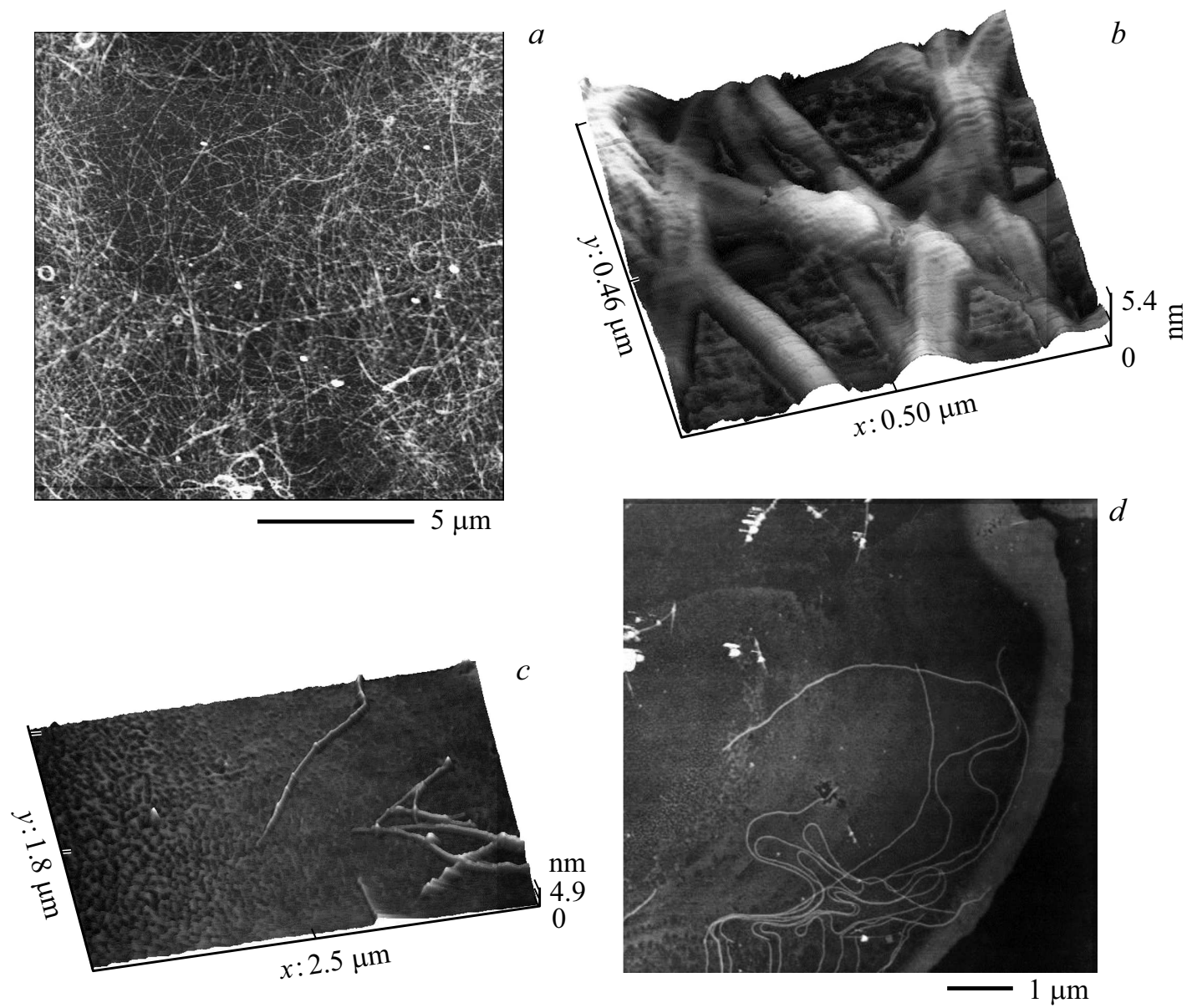

Рис. 3. Общее АСМ-изображение $10 \mathrm{~nm}$ тонкой ОУНТ-пленки (a), трехмерная поверхность $10 \mathrm{~nm}$ тонкой ОУНТ-пленки, полученная при помощи АCM $(b)$, трехмерная поверхность отдельных коротких ОУНТ- $(c)$ и АСМ-изображение отдельных длинных ОУНТ $(d)$.

ной моды изолированной ОУНТ на $\sim 10 \mathrm{~cm}^{-1}$. В исследовании [22] были найдены $c_{1}=215 \mathrm{~cm}^{-1} \cdot \mathrm{nm}$ и $c_{2}=18 \mathrm{~cm}^{-1}$. Таким образом, уравнение для диаметра $d$ ОУНТ будет иметь следующий вид

$$
d=\frac{c_{1}}{\omega_{R B M}-c_{2}} .
$$

Подставляя значение дыхательной моды отдельной ОУНТ $\left(144 \mathrm{~cm}^{-1}\right)$, а также значения $c_{1}$ и $c_{2}$ в уравнение (2) получим диаметр $d=1.706 \mathrm{~nm}$. Это значение попадает в ранее найденный диапазон значений диаметров тонких ОУНТ-пленок и согласуется со значениями, найденными в работе [23], для данного типа ОУНТ.

На спектрах КРС присутствуют линии, соответствующие так называемым $D\left(1346 \mathrm{~cm}^{-1}\right)$ и $G\left(1593 \mathrm{~cm}^{-1}\right)$ колебательным модам ОУНТ [20]. Отношение $G / D$ может быть использовано для анализа качества ОУНТ [24]. Большое значение $G / D$ говорит о малости дефектов в ОУНТ. Для нашего случая отношение $G / D$ было 18.71 . Такое значении $G / D$ сопоставимо со значениями высококачественных ОУНТ, полученных различными методами синтеза [23]. Например, отношения $G / D$ для НiPCO ОУНТ было 13.8, а для $A D$ ОУНТ было 52.2 [23].
Для исследования морфологии поверхности, а также для оценки длины ОУНТ в тонких пленках мы провели измерения поверхности с помощью атомно-силовой микроскопии (ACM). Для оценки длины ОУНТ был приготовлен образец с массовой концентрацией ОУНТ $\sim 2 \cdot 10^{-4 \%}$ в водной дисперсии. На рис. 2 . Представлены изображения поверхности тонкой ОУНТ-пленки, а также отдельных ОУНТ, полученных при помощи АСМ.

Как видно из рис. 3, $a$ тонкие ОУНТ-пленки имеют достаточно развитую поверхность, при этом образуются типичные пучки ОУНТ как показано на рис. $3, b$. Из pис. $3, c, d$ видно, что существуют отдельные ОУНТ c различной длиной. Некоторые ОУНТ имеют длину меньше $1 \mu \mathrm{m}$ (рис. $3, c)$. С другой стороны, как показано на рис. $2, d$, видны отдельные ОУНТ с сильно искривленной формой, где полная длина более $10 \mu \mathrm{m}$. В целом длина ОУНТ на подложке сильно зависит от способа приготовления дисперсий ОУНТ. Например, от времени и мощности ультразвуковой обработки дисперсий ОУНТ, которые используются для изготовления тонких пленок.

Известно, что углеродные материалы достаточно эффективно поглощают оптическое излучение [25]. По 

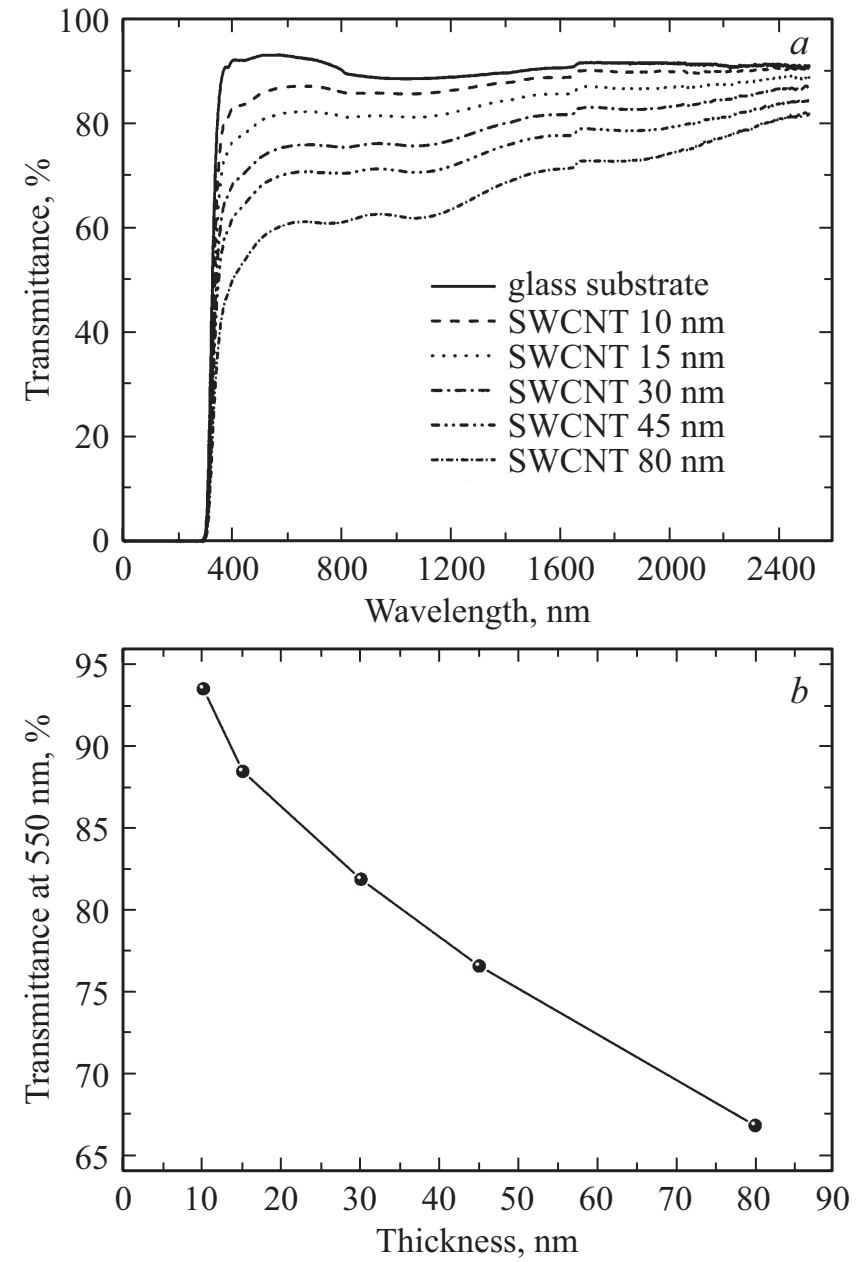

Рис. 4. Спектр пропускания тонких ОУНТ-пленок различной толщины на стеклянной подложке $(a)$, зависимость оптического пропускания на длине волны $550 \mathrm{~nm}$ от толщины тонкой ОУНТ-пленки $(b)$.

этой причине была измерена зависимость оптического пропускания от толщины тонких ОУНТ-пленок в диапазоне длин волн 200-2500 nm. Эта зависимость представлена на рис. 4, $a$. В общем случае ОУНТ являются эффективными широкозонными поглотителями света. Из рис. 4, $а$ видно, что спектр пропускания практически на всем диапазоне достаточно резко уменьшается при небольшом увеличении толщины тонкой пленки. Была построена зависимость пропускания на длине волны $550 \mathrm{~nm}$ от толщины тонкой ОУНТ-пленки (рис. 4,b). Здесь стоит отметить, что для зависимости пропускания тонких ОУНТ-пленок от толщины, представленной на рис. $3, b$, принималось $100 \%$ пропускание стеклянной подложки. Из рис. $4, a, b$ видно, что уже при толщинах более $15 \mathrm{~nm}$ пропускание становится ниже 85\%. Таким образом для создания оптически прозрачных тонких пленок на основе ОУНТ необходимо ограничиться толщиной порядка $15 \mathrm{~nm}$.

Для определения термоэлектрической добротности материалов важным является исследование электри- ческого сопротивления в зависимости от температуры. По этой причине было проведено исследование поверхностного сопротивления тонких ОУНТ пленок от комнатной температуры до $200^{\circ} \mathrm{C}$. Пересчет из поверхностного в удельное сопротивление производился с учетом всей толщины пленки. Здесь стоит отметить, что удельное сопротивление $10 \mathrm{~nm}$ тонкой ОУНТ-пленки было $\sim 3 \cdot 10^{-3} \mathrm{Ohm} \cdot \mathrm{cm}$ при комнатной температуре. С другой стороны, для 15, 30, 45 и $80 \mathrm{~nm}$ тонких ОУНТ-пленок удельное сопротивление было $\sim 1.5 \cdot 10^{-3}, \sim 1.8 \cdot 10^{-3}, \sim 1.7 \cdot 10^{-3}$ и $\sim 1.7 \cdot 10^{-3} \mathrm{Ohm} \cdot \mathrm{cm}$ соответственно. Возможно, что различия удельного сопротивления при малых толщинах связано с неплотной упаковкой ОУНТ по сравнению с более толстыми пленками.

Температурная зависимость поверхностного сопротивления для всех образцов тонких ОУНТ-пленок имела схожую тенденцию. На рис. 5 представлена типичная зависимость поверхностного сопротивления тонкой ОУНТ-пленки от температуры. Поверхностное сопротивление, как видно из рис. 5, возрастает при увеличении температуры. Такая зависимость отчасти характерна для металлов. В работах [26,27] наблюдалась схожая зависимость изменения поверхностного сопротивления тонких ОУНТ-пленок от температуры.

В ряде работ было показано, что электронные свойства ОУНТ являются чувствительными к молекулам кислорода и молекулам воды [28-30], которые находятся в окружающей среде. Возможно, что данные молекулы при комнатной температуре являются дополнительными акцепторами для электронов, которые есть в металлических ОУНТ. В основном электрическая проводимость в тонких ОУНТ-пленках осуществляется за счет дырок от полупроводниковых ОУНТ. Это связано с тем, что полупроводниковые ОУНТ составляют $\sim 2 / 3$ при синтезе ОУНТ [31]. Таким образом дополнительные акцепторы увеличивают электрическую проводимость в

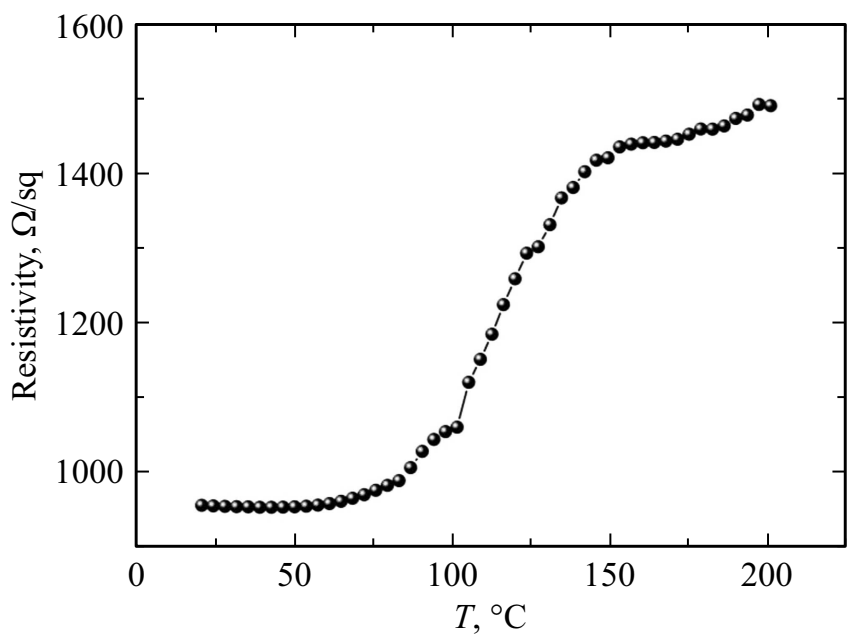

Рис. 5. Зависимость поверхностного сопротивления от температуры для $15 \mathrm{~nm}$ тонкой ОУНТ-пленки. 


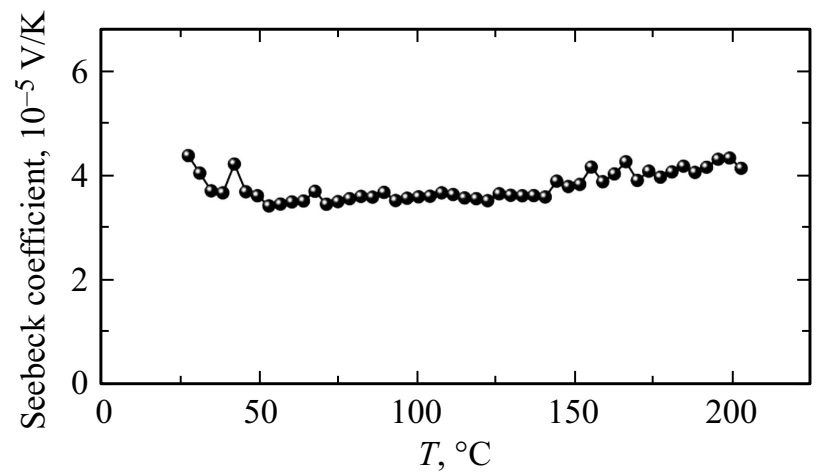

Рис. 6. Зависимость коэффициента Зеебека от температуры для $15 \mathrm{~nm}$ тонкой ОУНТ-пленки.

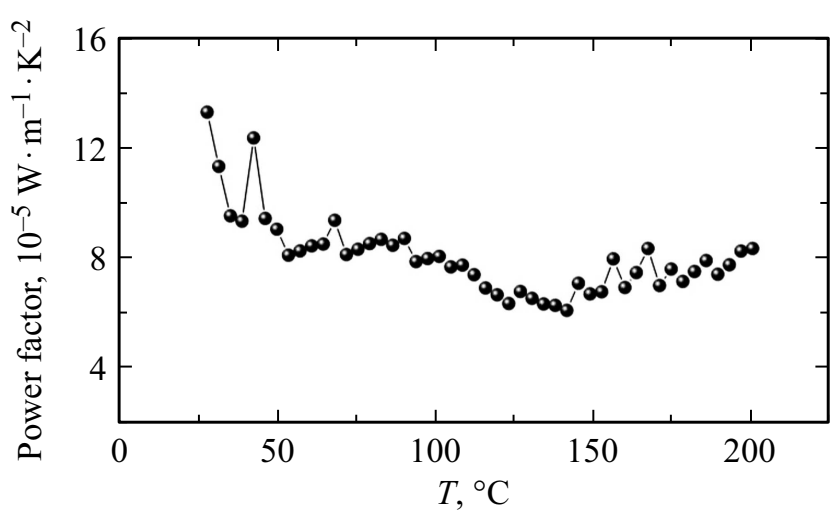

Рис. 7. Зависимость фактора мощности от температуры для $15 \mathrm{~nm}$ тонкой ОУНТ-пленки.

тонких ОУНТ-пленках. В свою очередь, возможно, при повышении температуры происходит десорбция молекул кислорода и молекул воды с поверхности тонких ОУНТ-пленок. В конечном результате уменьшается количество акцепторов и происходит уменьшение электрической проводимости за счет снижения концентрации дырок. Здесь стоит отметить, что вышеописанный возможный механизм требует отдельного экспериментального исследования.

На рис. 6 представлена температурная зависимость коэффициента Зеебека $15 \mathrm{~nm}$ тонкой ОУНТ-пленки. Все образцы тонких ОУНТ-пленок имели слабую зависимость коэффициента Зеебека от температуры, как показано на рис. 6. Из рис. 6 видно, что коэффициент Зеебека имеет значение $\sim 35-40 \mu \mathrm{V} / \mathrm{K}$ во всем температурном диапазоне. Стоит отметить, что для всех остальных образцов значение коэффициента Зеебека было $\sim 40 \mu \mathrm{V} / \mathrm{K}$. Более того, все измеренные образцы показывали дырочный тип проводимости согласно знаку коэффициента Зеебека. Полученные значения коэффициента Зеебека соответствуют диапазону значений, измеренных ранее для различных ОУНТ [31].

Одной из важных интегральных характеристик термоэлектрических материалов является фактор мощно- сти $P F\left(\mu \mathrm{W} \cdot \mathrm{m}^{-1} \cdot \mathrm{K}^{-2}\right)$, который определяется по следующей формуле:

$$
P F=S^{2} \sigma .
$$

Мы использовали формулу (3) чтобы построить температурную зависимость фактора мощности для $15 \mathrm{~nm}$ тонкой ОУНТ-пленки как показано на рис. 7.

Из рис. 7 видно, что фактор мощности $15 \mathrm{~nm}$ тонкой ОУНТ-пленки снижается при повышении температуры примерно до $140^{\circ} \mathrm{C} \mathrm{c} 120$ до $\sim 60 \mu \mathrm{W} \cdot \mathrm{m}^{-1} \cdot \mathrm{K}^{-2}$. Однако при дальнейшем увеличении температуры фактор мощности начинал слегка увеличиваться. По сравнению с другими ОУНТ, наши образцы тонких пленок показывали достаточно высокий фактор мощности [31].

Таким образом, представленные в настоящей работе исследования тонких ОУНТ-пленок могут быть использованы для разработки оптически прозрачных тонкопленочных термоэлектрических преобразователей на основе коммерчески доступных ОУНТ.

\section{4. Заключение}

Были изготовлены тонкие ОУНТ-пленки различной толщины из коммерчески доступных одностенных углеродных нанотрубок с помощью спрей-метода. Благодаря исследованиям просвечивающей электронной микроскопии в режиме съемки поперечного сечения были определены толщины тонких пленок. Толщина ОУНТ-пленок была 10, 15, 30, 45 и $80 \mathrm{~nm}$ соответственно. Из спектров КРС было выявлено, что диаметр ОУНТ лежит в диапазоне значений от $\sim 1.6$ до $\sim 1.8 \mathrm{~nm}$. Было установлено, что существуют ОУНТ с длиной более $10 \mu \mathrm{m}$.

С помощью спектров оптического пропускания было показано, что с увеличением толщины тонкой ОУНТ-пленки резко снижается пропускание. Оптимальная толщина тонкой пленки, при которой пропускание имеет значение более 85

Было установлено, что удельное сопротивление $10 \mathrm{~nm}$ тонкой ОУНТ-пленки было $\sim 3 \cdot 10^{-3} \mathrm{Ohm} \cdot \mathrm{cm}$ при комнатной температуре. С другой стороны, для 15, 30, 45 и $80 \mathrm{~nm}$ тонких ОУНТ-пленок удельное сопротивление было $\sim 1.5 \cdot 10^{-3}, \sim 1.8 \cdot 10^{-3}, \sim 1.7 \cdot 10^{-3}$ и $\sim 1.7 \cdot 10^{-3} \mathrm{Ohm} \cdot \mathrm{cm}$ соответственно. Возможно, что различные значения удельного сопротивления при малых толщинах связано с неплотной упаковкой ОУНТ по сравнению с более толстыми пленками. Обнаружено, что поверхностное сопротивление возрастает при увеличении температуры. Возможно, это связано с температурно-индуцированной адсорбцией молекул кислорода на поверхности тонких ОУНТ-пленок.

Тонкие ОУНТ-пленки имели слабую зависимость коэффициента Зеебека от температуры. Все образцы имели значение коэффициента Зеебека $\sim 40 \mu \mathrm{V} / \mathrm{K}$. Тонкие пленки показывали дырочный тип проводимости согласно знаку коэффициента Зеебека. Было установлено, что фактор мощности $15 \mathrm{~nm}$ тонкой ОУНТ-пленки снижается при повышении температуры примерно до 
$140^{\circ} \mathrm{C}$ c $\sim 120$ до $\sim 60 \mu \mathrm{W} \cdot \mathrm{m}^{-1} \cdot \mathrm{K}^{-2}$. При дальнейшем увеличении температуры фактор мощности начинал увеличиваться.

Исследования тонких ОУНТ-пленок могут быть полезны для разработки оптически прозрачных тонкопленочных термоэлектрических преобразователей на основе коммерчески доступных ОУНТ.

\section{Список литературы}

[1] N. Toshima. Synthetic Met. 225, 3 (2017).

[2] G.J. Snyder, E.S. Toberer. Nature Mater. 7, 105 (2008).

[3] X. Mu, H.Y. Zhou, D.Q. He, W.Y. Zhao, P. Wei, W.T. Zhu, X.L. Nie, H.J. Liu, Q.J. Zhang. Nano Energy 33, 55 (2017).

[4] D. Madan, Z.Q. Wang, P.K. Wright, J.W. Evans. Appl Energ. 156, 587 (2015).

[5] Y.N. Chen, Y. Zhao, Z.Q. Liang. Energ Environ Sci. 8, 401 (2015).

[6] J.F. Li, W.S. Liu, L.D. Zhao, M. Zhou. Npg Asia Mater. 2, 152 (2010).

[7] J. He, T.M. Tritt. Science 357, 1369 (2017).

[8] X.L. Su, P. Wei, H. Li, W. Liu, Y.G. Yan, P. Li, C.Q. Su, C.J. Xie, W.Y. Zhao, P.C. Zhai, Q.J. Zhang, X.F. Tang, C. Uher. Adv. Mater. 29, 1602013 (2017).

[9] S. Ortega, M. Ibanez, Y. Liu, Y. Zhang, M.V. Kovalenko, D. Cadavid, A. Cabot. Chem. Soc. Rev. 46, 3510 (2017).

[10] L.D. Hicks, M.S. Dresselhaus. Phys. Rev. B 47, 12727 (1993).

[11] K. Yanagi, S. Kanda, Y. Oshima, Y. Kitamura, H. Kawai, T. Yamamoto, T. Takenobu, Y. Nakai, Y. Maniwa. Nano Lett. 14, 6437 (2014).

[12] Y. Nakai, K. Honda, K. Yanagi, H. Kataura, T. Kato, T. Yamamoto, Y. Maniwa. Appl. Phys. Exp. 7, 025103 (2014).

[13] A.P. Tsapenko, A.E. Goldt, E. Shulga, Z.I. Popov, K.I. Maslakov, A.S. Anisimov, P.B. Sorokin, A.G. Nasibulin. Carbon 130, 448 (2018).

[14] C. Yang, D. Souchay, M. Kneiss, M. Bogner, M. Wei, M. Lorenz, O. Oeckler, G. Benstetter, Y.Q. Fu, M. Grundmann. Nature Commun. 8, 16076 (2017).

[15] I.A. Tambasov, A.S. Tarasov, M.N. Volochaev, M.V. Rautskii, V.G. Myagkov, L.E. Bykova, V.S. Zhigalov, A.A. Matsynin, E.V. Tambasova. Physica E 84, 162 (2016).

[16] V.G. Myagkov, L.E. Bykova, A.A. Matsynin, M.N. Volochaev, V.S. Zhigalov, I.A. Tambasov, Y.L. Mikhlin, D.A. Velikanov, G.N. Bondarenko. J. Solid State Chem. 246, 379 (2017).

[17] M.S. Dresselhaus, G. Dresselhaus, A. Jorio, A.G. Souza, R. Saito. Carbon 40, 2043 (2002).

[18] T. Belin, F. Epron. Mater. Sci. Eng. B 119, 105 (2005).

[19] M.S. Dresselhaus, A. Jorio, M. Hofmann, G. Dresselhaus, R. Saito. Nano Lett. 10, 751 (2010).

[20] M.S. Dresselhaus, P.C. Eklund. Adv. Phys. 49, 705 (2000).

[21] L. Henrard, V.N. Popov, A. Rubio. Phys. Rev. B 64, 205403 (2001).

[22] J. Maultzsch, H. Telg, S. Reich, C. Thomsen. Phys. Rev. B 72, 205438 (2005).

[23] A. Chortos, I. Pochorovski, P. Lin, G. Pitner, X.Z. Yan, T.Z. Gao, J.W.F. To, T. Lei, J.W. Will, H.S.P. Wong, Z.N. Bao. Acs Nano 11, 5660 (2017).

[24] V.M. Irurzun, M.P. Ruiz, D.E. Resasco. Carbon 48, 2873 (2010).
[25] A.A. Ivanenko, I.A. Tambasov, A.A. Pshenichnaia, N.P. Shestakov. Opt. Mater. 73, 388 (2017).

[26] B.B. Parekh, G. Fanchini, G. Eda, M. Chhowalla. Appl. Phys. Lett. 90, 121913 (2007).

[27] D. Kim, H.C. Lee, J.Y. Woo, C.S. Han. J. Phys. Chem. C 114, 5817 (2010).

[28] P.S. Na, H.J. Kim, H.M. So, K.J. Kong, H.J. Chang, B.H. Ryu, Y.M. Choi, J.O. Lee, B.K. Kim, J.J. Kim, J.H. Kim. Appl. Phys. Lett. 87, 093101 (2005).

[29] P.G. Collins, K. Bradley, M. Ishigami, A. Zettl. Science 287, 1801 (2000)

[30] A. Zahab, L. Spina, P. Poncharal, C. Marliere. Phys. Rev. B 62, 10000 (2000).

[31] J.L. Blackburn, A.J. Ferguson, C. Cho, J.C. Grunlan. Adv. Mater. 30, 1704386 (2018)

Редактор Т.Н. Василевская 